\title{
The rise of Newtonian drops in a nematic liquid crystal
}

\author{
CHUNFENG ZHOU ${ }^{1}$, PENGTAO YUE Y $^{1,2}$ \\ AND JAMES J. FEN G ${ }^{1,2}$ \\ ${ }^{1}$ Department of Chemical and Biological Engineering, University of British Columbia, Vancouver, \\ BC V6T 1Z3, Canada \\ ${ }^{2}$ Department of Mathematics, University of British Columbia, Vancouver, BC V6T 1Z2, Canada
}

(Received 11 February 2007 and in revised form 21 August 2007)

We simulate the rise of Newtonian drops in a nematic liquid crystal parallel to the far-field molecular orientation. The moving interface is computed in a diffuseinterface framework, and the anisotropic rheology of the liquid crystal is represented by the Leslie-Ericksen theory, regularized to permit topological defects. Results reveal interesting coupling between the flow field and the orientational field surrounding the drop, especially the defect configuration. The flow generally sweeps the point and ring defects downstream, and may transform a ring defect into a point defect. The stability of these defects and their transformation are depicted in a phase diagram in terms of the Ericksen number and the ratio between surface anchoring and bulk elastic energies. The nematic orientation affects the flow field in return. Drops with planar anchoring on the surface rise faster than those with homeotropic anchoring, and the former features a vortex ring in the wake. These are attributed to the viscous anisotropy of the nematic. With homeotropic anchoring, the drop rising velocity experiences an overshoot, owing to the transformation of the initial surface ring defect to a satellite point defect. With both types of anchoring, the drag coefficient of the drop decreases with increasing Ericksen number as the flow-alignment of the nematic orientation reduces the effective viscosity of the liquid crystal.

\section{Introduction}

Nematic liquid crystals exhibit special electro-optical properties and find applications in numerous modern technologies. As complex fluids, they are distinguished microscopically by molecular alignment and long-range orientation order, and macroscopically by a liquid-solid duality in that they flow as anisotropic viscous fluids but resist orientational distortion as elastic solids (de Gennes \& Prost 1993). In a fluid-mechanical context, the motion of a particle or drop in a nematic is of fundamental interest, being the counterpart of the Stokes or Hadamard-Rybczynski problem in viscous Newtonian fluids. Besides, suspensions and emulsions in nematic matrices show intriguing mesoscopic structures and mechanical properties that suggest new applications (Poulin, Stark, Lubensky \& Weitz 1997b; Poulin \& Weitz 1998; Loudet, Barois \& Poulin 2000; Tixier, Heppenstall-Butler \& Terentjev 2006).

Particle motion in nematic liquid crystals is much more complex than the Stokes problem. Even in a static nematic, insertion of a particle or drop normally causes the nucleation of orientational defects (Poulin et al. 1997b; Lavrentovich 1998; Feng \& Zhou 2004). The liquid crystal molecules prefer a certain orientation on interfaces, 
the most common being homeotropic (normal) and planar (tangential) anchoring. If the orientation field surrounding the drop or particle comes into conflict with the far-field orientation, defects form. These may be seen as singularities in the director field $\boldsymbol{n}(\boldsymbol{r})$, which represents the average molecular orientation at each spatial point. For a particle with homeotropic anchoring, experiments have recorded two types of defects: a 'satellite' point defect (Poulin et al. 1997b; Poulin \& Weitz 1998; Lubensky et al. 1998) and a 'Saturn-ring' line defect that encircles the particle on its equator (Mondain-Monval et al. 1999; Gu \& Abbott 2000). With planar anchoring, two surface defects known as 'boojums' form at the poles (Poulin \& Weitz 1998). Orientational defects have long been an important subject of liquid crystal physics and indeed condensed matter physics in general (Trebin 1982; Kléman 1983).

For moving particles, the earliest studies were falling-ball experiments to measure the effective viscosity of liquid crystals (White, Cladis \& Torza 1977; Kuss 1978). More recently, Poulin et al. (1997a) used the 'Stokes drag' to verify the dipolar attraction force between two droplets in a nematic fluid. In such dynamic situations, the flow modifies the director field and defect configuration near the particle. The latter in turn affect the rheology of the liquid crystal and thus the flow field. Therefore, the two-way coupling between flow and microstructure is the key physics governing particle motion in nematics. In general, such coupling has been formulated by constitutive theories for nematic liquid crystals (de Gennes \& Prost 1993; Rey \& Tsuji 1998; Feng, Sgalari \& Leal 2000). Owing to the rheological complexity, only a handful of theoretical studies have appeared on the moving particle problem, most of which sought to decouple the flow field and the director field (Stark 2001). For instance, the director field may be fixed at the static solution, and the resulting flow field and drag are calculated (Ruhwandl \& Terentjev 1996; Stark \& Ventzki 2001). This corresponds to the low Ericksen number $(E r)$ limit, where the viscous forces are too weak to modify the orientational field maintained by elasticity. Conversely, the Newtonian flow field may be prescribed, and the director field $\boldsymbol{n}(\boldsymbol{r})$ is calculated as a result (Diogo 1983; Yoneya et al. 2005). This may be linked to the high-Er limit. Stark \& Ventzki (2002) seem to have been the first to tackle the flow-director two-way coupling at finite $E r$. In flow around a sphere with a satellite point defect, they predicted a counter-intuitive flow effect that moves the defect upstream. This was contradicted by Yoneya et al. (2005) who showed that the defect shifts downstream at a similar Ericksen number. But the latter study prescribed the Stokes flow field, and it is unclear whether the decoupling accounts for the discrepancy. To our knowledge, the only other coupled study is Fukuda et al. (2004), who showed that the flow tends to convect the $\boldsymbol{n}$ field downstream, while $\boldsymbol{n}$ modifies the velocity field and makes it fore-aft asymmetric. Unfortunately, Fukuda et al. assumed an isotropic viscosity and thus omitted an important component of the liquid crystal rheology. Therefore, a 'complete solution' that fully couples flow and director fields and incorporates viscous-elastic duality and anisotropy is not yet available.

Most prior calculations assumed rigid anchoring on the particle surface. In reality, the anchoring strength is finite, representable by an anchoring energy, and has a major role in defining the defect configuration (Lubensky et al. 1998; Mondain-Monval et al. 1999). Furthermore, only solid particles have been considered in theoretical studies so far, even though most of the experimental observations have come from emulsions with isotropic droplets suspended in nematics (Poulin \& Weitz 1998). If the interfacial tension is not so strong as to overwhelm the surface anchoring, the interplay between the two is known to lead to unique drop and bubble shapes (Nastishin et al. 2005; Akers \& Belmonte 2006; Zhou et al. 2007). These lacunae in our current 
understanding have motivated the present simulations based on the Leslie-Ericksen theory.

Simulating the rise of Newtonian drops in a nematic liquid crystal is a computational challenge because of the well-known numerical difficulties in handling moving and deforming interfaces as well as the complex rheology of the nematic liquid crystal. Not only are the rheological properties and stresses discontinuous across the interface, they are anisotropic and evolving with the microstructure in the nematic component. In principle, the balance between the stresses and the surface tension determines the motion of the interface, which must be tracked dynamically on a moving grid while solving for the flow in each component. As an alternative, we have developed an energy-based diffuse-interface method that handles both the interface and the rheology in a unified framework (Yue et al. 2004; Feng et al. 2005). The interface is now a thin diffuse layer defined by a phase-field variable. A mixing energy governs the interaction of the two components. As long as the microstructure of the complex fluid is describable by a free energy, as is the case for liquid crystals, that energy can be combined with the mixing energy to form the total free energy of the system. A formal variational procedure then leads to the proper governing equations of the two-fluid system. To solve these, Yue et al. (2006b) have developed two-dimensional and axisymmetric finite-element algorithms based on adaptive mesh generation, which is key to resolving the thin interface. The method has proved accurate and efficient in simulating dynamics of viscoelastic drops and jets (Yue et al. 2005d; Yue, Zhou \& Feng 2006a; Zhou et al. 2006), and will be adapted to the problem at hand.

This study has three objectives: (1) to demonstrate that the motion of Newtonian drops in a nematic fluid can be successfully simulated by our diffuse-interface method, incorporating complex rheology, deformable interfaces and a variable anchoring strength; (2) to investigate how flow modifies the orientational field and especially the defect configuration near the drop; and (3) to investigate how the director field modifies the flow in return, especially how the rheological anisotropy affects the rising velocity of the drops and the drag force on them.

\section{Theory and numerical method}

Yue et al. (2004, 2006b) have described the theoretical model and the numerical method in detail, and validated the methodology by benchmark problems. Planar two-dimensional and axisymmetric applications to drop dynamics have been reported recently (Yue et al. 2005a,b,c,d, 2006a; Zhou, Yue \& Feng 2006). Therefore, we will only summarize the main ideas and give the governing equations for a two-component mixture of a Newtonian fluid and a nematic liquid crystal. The diffuse interface has a small but non-zero thickness, inside which the two components are mixed and store a mixing energy. We define a phase-field variable $\phi$ such that the concentrations of the nematic and Newtonian components are $(1+\phi) / 2$ and $(1-\phi) / 2$, respectively. Then $\phi=1$ in the bulk nematic phase, and $\phi=-1$ in the bulk Newtonian phase. The interface is taken to be the level set $\phi=0$. There are three types of free energies in this system: mixing energy of the interface, bulk distortion energy of the nematic, and the anchoring energy of the liquid crystal molecules on the interface:

$$
\begin{aligned}
& f_{\text {mix }}=\frac{\lambda}{2}\left[|\nabla \phi|^{2}+\frac{\left(\phi^{2}-1\right)^{2}}{2 \epsilon^{2}}\right], \\
& f_{\text {bulk }}=\frac{K}{2}\left[\nabla \boldsymbol{n}:(\nabla \boldsymbol{n})^{T}+\frac{\left(|\boldsymbol{n}|^{2}-1\right)^{2}}{2 \delta^{2}}\right],
\end{aligned}
$$




$$
f_{\text {anch }}= \begin{cases}\frac{A}{2}(\boldsymbol{n} \cdot \nabla \phi)^{2} & \text { (planar anchoring) } \\ \frac{A}{2}\left[|\boldsymbol{n}|^{2}|\nabla \phi|^{2}-(\boldsymbol{n} \cdot \nabla \phi)^{2}\right] & \text { (homeotropic anchoring). }\end{cases}
$$

In $f_{\text {mix }}, \lambda$ is the mixing energy density, $\epsilon$ is the capillary width and the ratio $2 \sqrt{2} \lambda / 3 \epsilon$ produces the interfacial tension $\sigma$ (Jacqmin 1999; Liu \& Shen 2003; Yue et al. 2004); $f_{\text {bulk }}$ is the Frank energy with a single elastic constant $K$. Different elastic constants may be assigned to different modes of distortion (de Gennes \& Prost 1993), but we use the one-constant approximation for simplicity. Note that $f_{\text {bulk }}$ is regularized to permit defects where $|\boldsymbol{n}|$ deviates from unity over the defect core of size $\delta$. The second term, devised by Liu \& Walkington (2000) after the Ginzburg-Landau energy of (2.1), represents the distortion energy of the defect by an energy penalty against the shortening of $|\boldsymbol{n}|$, effectively using $|\boldsymbol{n}|$ as an order parameter. For $f_{\text {anch }}$, we adapt the Rapini-Papoular form (Rapini \& Papoular 1969) to our diffuse-interface framework, with $A$ being the anchoring energy density and $W=2 \sqrt{2} A / 3 \epsilon$ giving the surface anchoring strength (Yamamoto 2001). Now we have the total free energy density for the two-phase material:

$$
f(\phi, \boldsymbol{n}, \nabla \boldsymbol{\phi}, \nabla \boldsymbol{n})=f_{m i x}+\frac{1+\phi}{2} f_{b u l k}+f_{\text {anch }} .
$$

A variation on the free energy, supplemented by the various dissipative terms, leads to the following governing equations for the configuration variables $\boldsymbol{v}, p, \phi$ and $\boldsymbol{n}$ (Yue et al. 2004):

$$
\begin{aligned}
\nabla \cdot \boldsymbol{v} & =0 \\
\rho\left(\frac{\partial \boldsymbol{v}}{\partial t}+\boldsymbol{v} \cdot \nabla \boldsymbol{v}\right) & =-\nabla p+\nabla \cdot \boldsymbol{\sigma}-\rho g \boldsymbol{e}_{z}, \\
\frac{\partial \phi}{\partial t}+\boldsymbol{v} \cdot \nabla \phi & =\gamma \lambda \nabla^{2}\left[-\nabla^{2} \phi+\frac{\phi\left(\phi^{2}-1\right)}{\epsilon^{2}}\right], \\
\boldsymbol{h} & =\gamma_{1} \boldsymbol{N}+\gamma_{2} \boldsymbol{D} \cdot \boldsymbol{n} .
\end{aligned}
$$

The density $\rho$ is the average between the nematic density $\rho_{1}$ and the Newtonian density $\rho_{2}$ :

$$
\rho=\frac{1+\phi}{2} \rho_{1}+\frac{1-\phi}{2} \rho_{2},
$$

$g$ is the gravitational acceleration and $\boldsymbol{e}_{z}$ is the upward unit vector. The phase-field variable $\phi$ obeys the Cahn-Hilliard equation, $\gamma$ being the mobility parameter of the diffuse interface (Yue et al. 2004; Yue, Zhou \& Feng 2007). The deviatoric stress tensor

$$
\boldsymbol{\sigma}=-\lambda(\nabla \phi \otimes \nabla \phi)-K \frac{1+\phi}{2}(\nabla \boldsymbol{n}) \cdot(\nabla \boldsymbol{n})^{T}-\boldsymbol{G}+\frac{1+\phi}{2} \boldsymbol{\sigma}^{\prime}+\frac{1-\phi}{2} \mu\left[\nabla \boldsymbol{v}+(\nabla \boldsymbol{v})^{T}\right],
$$

with $\boldsymbol{G}=A(\boldsymbol{n} \cdot \nabla \phi) \boldsymbol{n} \otimes \nabla \phi$ for planar anchoring and $\boldsymbol{G}=A[(\boldsymbol{n} \cdot \boldsymbol{n}) \nabla \boldsymbol{\phi}-(\boldsymbol{n} \cdot \nabla \phi) \boldsymbol{n}] \otimes \nabla \phi$ for homeotropic anchoring, and $\mu$ being the viscosity of the Newtonian component; $\boldsymbol{\sigma}^{\prime}$ is the Leslie viscous stress in the nematic phase (Leslie 1968),

$$
\boldsymbol{\sigma}^{\prime}=\alpha_{1} \boldsymbol{D}: \boldsymbol{n n n n}+\alpha_{2} \boldsymbol{n} \boldsymbol{N}+\alpha_{3} \boldsymbol{N n}+\alpha_{4} \boldsymbol{D}+\alpha_{5} \boldsymbol{n n} \cdot \boldsymbol{D}+\alpha_{6} \boldsymbol{D} \cdot \boldsymbol{n n},
$$

where $\alpha_{1}$ to $\alpha_{6}$ are the Leslie viscous coefficients observing the Onsager relationship (de Gennes \& Prost 1993): $\alpha_{2}+\alpha_{3}=\alpha_{6}-\alpha_{5} . \boldsymbol{D}=(1 / 2)\left[\nabla \boldsymbol{v}+(\nabla \boldsymbol{v})^{T}\right]$ is the strain 
rate tensor, and $\boldsymbol{N}=(\mathrm{d} \boldsymbol{n} / \mathrm{d} t)-(1 / 2)\left[(\nabla v)^{T}-\nabla \boldsymbol{v}\right] \cdot \boldsymbol{n}$ is the rotation of the director $\boldsymbol{n}$ with respect to the background flow field. The $\boldsymbol{n}$ field evolves in the flow according to a balance between elastic and viscous torques as given in (2.8). The elastic torque is represented by the molecular field (de Gennes \& Prost 1993):

$$
\boldsymbol{h}=K\left[\nabla \cdot\left(\frac{1+\phi}{2} \nabla \boldsymbol{n}\right)-\frac{1+\phi}{2} \frac{\left(\boldsymbol{n}^{2}-1\right) \boldsymbol{n}}{\delta^{2}}\right]-\boldsymbol{g},
$$

with $\boldsymbol{g}=A(\boldsymbol{n} \cdot \nabla \phi) \nabla \phi$ for planar anchoring, and $\boldsymbol{g}=A[((\nabla \phi \cdot \nabla \phi) \boldsymbol{n}-(\boldsymbol{n} \cdot \nabla \phi) \nabla \phi]$ for homeotropic anchoring. Both $\boldsymbol{g}$ and $\boldsymbol{G}$ derive from the anchoring energy $f_{\text {anch }}$ through a variational procedure (Yue et al. 2004). The coefficients $\gamma_{1}=\alpha_{3}-\alpha_{2}$ and $\gamma_{2}=\alpha_{3}+\alpha_{2}$.

Obviously, the apparent viscosity of the nematic depends on the orientation of $\boldsymbol{n}$ relative to the flow. This viscous anisotropy is commonly represented by the Miesowicz viscosities in a simple shear flow (de Gennes \& Prost 1993):

$$
\begin{aligned}
& \eta_{1}=\frac{1}{2}\left(-\alpha_{2}+\alpha_{4}+\alpha_{5}\right), \\
& \eta_{2}=\frac{1}{2}\left(\alpha_{3}+\alpha_{4}+\alpha_{6}\right),
\end{aligned}
$$

which are measured with $\boldsymbol{n}$ held perpendicular and parallel to the flow direction, respectively; $\eta_{1}>\eta_{2}$. If $\boldsymbol{n}$ makes an angle $\theta$ with the flow, the general formula for the shear viscosity is (Carlsson 1984):

$$
\eta(\theta)=\eta_{2}-\left(\alpha_{2}+\alpha_{3}\right) \sin ^{2} \theta .
$$

A third Miesowicz viscosity may be defined with $\boldsymbol{n}$ along the vorticity axis. This is irrelevant to the present study which constrains $\boldsymbol{n}$ to the meridional plane in axisymmetric geometries. Without external fields or wall anchoring, $\boldsymbol{n}$ achieves a steady alignment in simple shear if $\alpha_{2} / \alpha_{3}>0$, but tumbles endlessly if the ratio is negative. Most liquid crystals are of the aligning type (de Gennes \& Prost 1993), and the distinction is insignificant in complex flow fields as simulated here. Thus, we have used $\alpha$ values based on the aligning PAA and MBBA in the rest of this paper.

To arrive at the Cahn-Hilliard equation (2.7), we have omitted from the right-hand side coupling terms between $f_{m i x}$ and the nematic energies. These are insignificant as long as the interface stays narrow. In fact, the Cahn-Hilliard diffusive dynamics has a visible effect only during singular events such as film rupture (Yue et al. 2005a). In the current context, the diffuse interface may be seen as merely a numerical device for treating a moving internal boundary.

The governing equations are solved, in axisymmetric geometries, by a numerical scheme AMPHI that employs Galerkin finite elements with adaptive meshing (Yue et al. 2006b). The latter has proved to be key to accurate phase-field simulations, and the interface of thickness $O(4 \epsilon)$ requires roughly 10 grid points to resolve (Feng et al. 2005). In addition, the defect regions are covered with fine grids as well (see figure $3 b$ ). Since the size of the defect core is comparable to the interfacial thickness (Stark 1999), $\delta$ is chosen to be $4 \epsilon$. We use implicit time-stepping, with the time step automatically adjusted according to the motion of the interface. Numerical experiments with grid refinement and time-step refinement have been carried out (Yue et al. 2006b), and adequate resolution is ensured for the simulations presented in the following. 


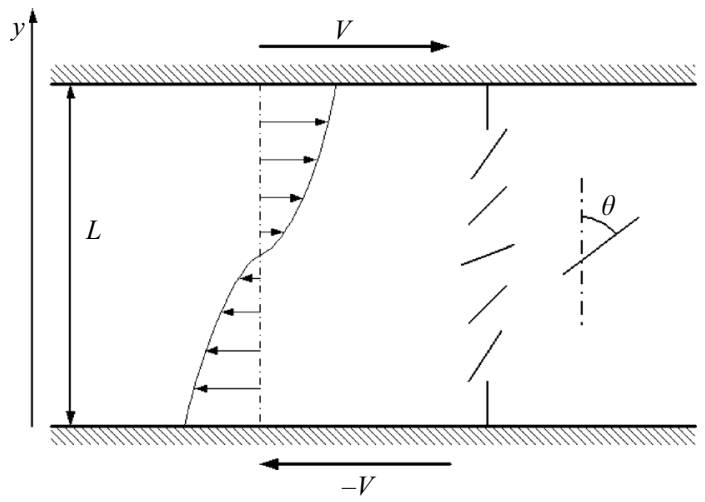

FIGURE 1. Simple shear flow of a nematic with homeotropic anchoring on the walls. The director orientation is indicated by $\theta(y)$, and the velocity $v(y)$ deviates from a linear profile because of the anisotropic viscosity.

\section{Results and discussion}

\subsection{Simple shear flow as validation}

Consider the simple shear flow of a nematic between parallel walls in figure 1, with rigid homeotropic anchoring on the walls. A one-dimensional analytical solution is available if $\alpha_{1}$ vanishes, and we will compute the same flow in a two-dimensional domain and use the exact solution to validate our numerical treatment of the LeslieEricksen theory. In the one-dimensional solution due to Carlsson (1984), the velocity profile $v(y)$ and orientation profile $\theta(y)$ are given by coupled equations:

$$
\begin{aligned}
v(y) & =\int_{0}^{y} \frac{\tau}{\eta_{2}-\left(\alpha_{2}+\alpha_{3}\right) \cos ^{2} \theta(y)} \mathrm{d} y-V, \\
y(\theta) & =\frac{1}{\sqrt{2 \tau}} \int_{0}^{\theta} \frac{\sqrt{K}}{\sqrt{F(\theta)-F\left(\theta_{m}\right)}} \mathrm{d} \theta, \\
\tau & =\frac{2}{L^{2}}\left[\int_{0}^{\theta_{m}} \frac{\sqrt{K}}{\sqrt{F(\theta)-F\left(\theta_{m}\right)}}\right]^{2}, \\
F(\theta) & =\left(\frac{\alpha_{2}}{\sqrt{\eta_{1} \eta_{2}}}+\sqrt{\frac{\eta_{1}}{\eta_{2}}}\right) \tan ^{-1}\left(\sqrt{\frac{\eta_{2}}{\eta_{1}}} \tan \theta\right)-\theta,
\end{aligned}
$$

where $\tau$ is the constant shear stress on the plates determined from $\theta_{m}=\theta(L / 2)$, and $\theta_{m}$, the largest rotation angle at the centre between the walls, is in turn determined by the condition $V=v(L)$. This solution assumes $\alpha_{1}=0$, and is written in a slightly simplified form here because of the one-constant approximation.

Our two-dimensional computation uses a domain of length $5 \mathrm{~L}$ divided into 8235 triangular elements. Figure 2 compares our solution with Carlsson's analytical solution, and the two are in excellent agreement. Note that the maximum director angle $\theta_{m}=77.5^{\circ}$ is short of the Leslie angle $\theta_{0}=80^{\circ}$ because at $E r=\bar{\eta} V L / K=35$, the viscous effect is not strong enough to completely dominate the elastic effect. In $E r$, the characteristic viscosity of the nematic is taken to be $\bar{\eta}=\left(\eta_{1}+\eta_{2}\right) / 2=\left(\alpha_{3}+\alpha_{4}+\alpha_{5}\right) / 2$. The rotation of $\boldsymbol{n}$ into the flow direction reduces the local viscosity, and the velocity profile $v(y)$ reacts by diminishing the shear rate at the walls and increasing it in the 
(a)

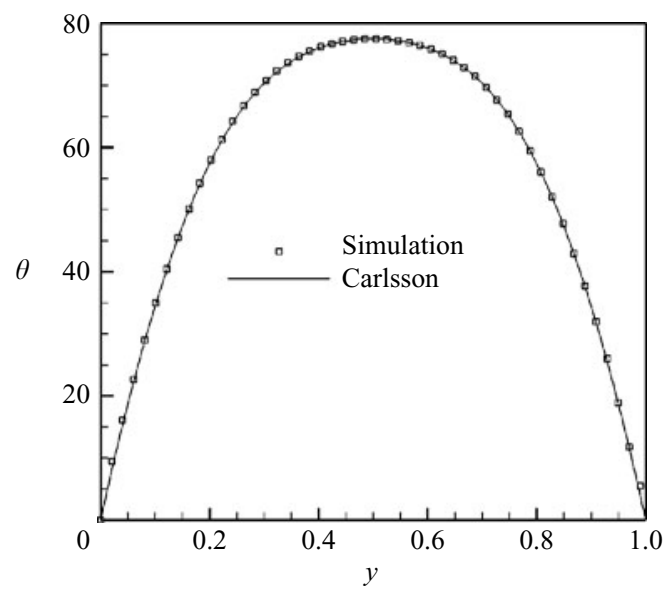

(b)

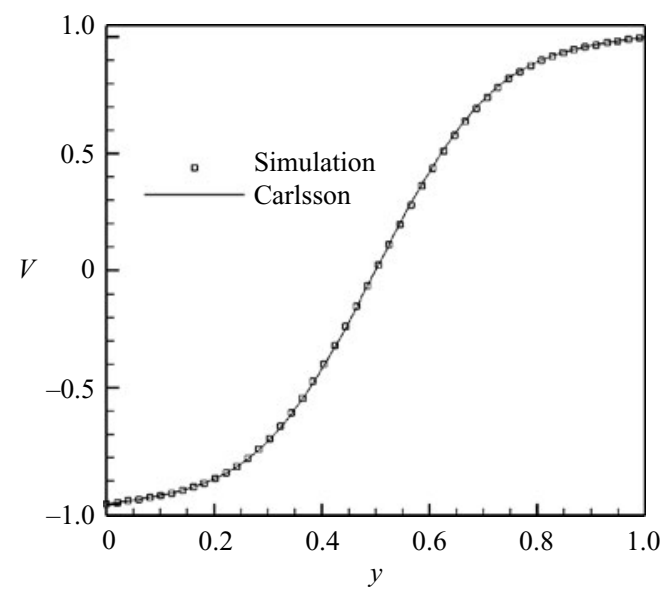

FiguRE 2. Comparison between our results and the one-dimensional exact solution of Carlsson (1984). (a) The director orientation profile; $(b)$ the velocity profile. The Leslie coefficients are $\alpha_{1}=0, \alpha_{2} / \alpha_{4}=-1.78, \alpha_{3} / \alpha_{4}=-0.056, \alpha_{5} / \alpha_{4}=1, \alpha_{6} / \alpha_{4}=-0.83$, and the Leslie angle $\theta_{0}=\tan ^{-1} \sqrt{\alpha_{2} / \alpha_{3}}=80^{\circ}$. The flow velocity corresponds to an Ericksen number $\mathrm{Er}=35$.

centre to maintain a constant shear stress. In this case, the minimum viscosity at the centre $\eta_{m}$ is such that $\eta_{1} / \eta_{m}=13.4$ and $\eta_{m} / \eta_{2}=2.55$.

\subsection{Static orientational defects}

When a drop has planar anchoring on its surface, boojums are the only possible defects, even when the drop is moving in the liquid crystal. With homeotropic anchoring, on the other hand, multiple defect patterns may appear and interesting transformations take place. Thus we will only consider homeotropic anchoring in this subsection, examining defects surrounding stationary particles as a preface to the flow-induced transformation discussed in the next subsection. A more or less coherent picture has emerged about defects near a stationary particle (Ruhwandl \& Terentjev 1997; Stark 2001; Feng \& Zhou 2004). The Saturn ring and the satellite point defect are the two possible configurations (figures $3 a$ and $3 c$ ), and their stability depends on the relative importance of surface anchoring and bulk elasticity, represented by the dimensionless group

$$
A_{K}=\frac{W a}{K},
$$

$a$ being the effective radius of the drop. A Saturn ring incurs more distortion to the surface anchoring while a satellite costs more bulk energy. Thus, rings are favoured at smaller $A_{K}$. Indeed, the point defect becomes unstable below a critical $A_{K}$, and spontaneously opens into a Saturn ring. For sufficiently weak anchoring, the Saturn ring shrinks onto the particle surface or even into the particle as an imaginary ring' (Kuksenok et al. 1996). For larger $A_{K}$, both point and ring defects are stable, and either can be realized from proper initial conditions. The point defect becomes energetically more favourable with increasing $A_{K}$, but it is unclear whether the ring ever becomes unstable (Ruhwandl \& Terentjev 1997; Feng \& Zhou 2004).

In regularizing the Leslie-Ericksen theory to allow defects (2.2), we treat $\boldsymbol{n}(\boldsymbol{r})$ as a vector field. In reality, the molecular orientation is a pseudo-vector that does 
(a)

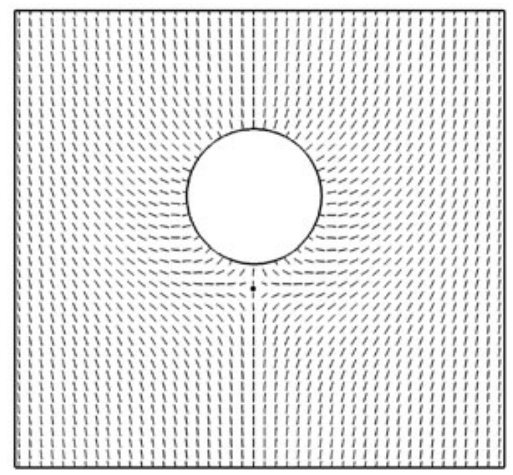

(c)

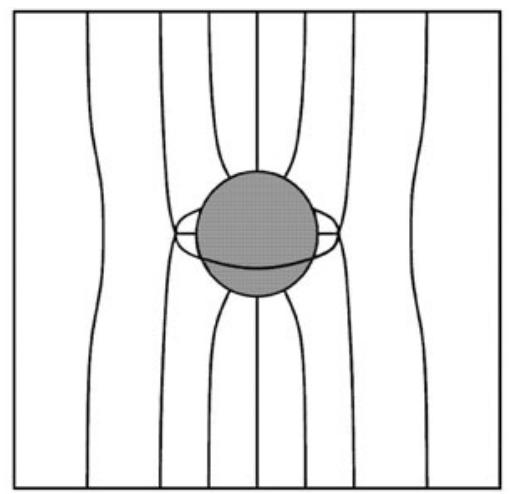

(b)

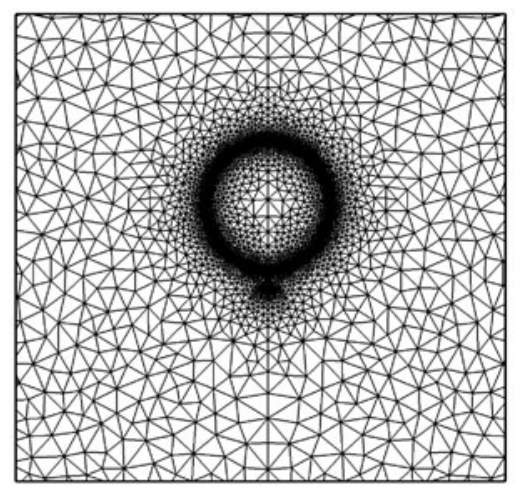

(d)

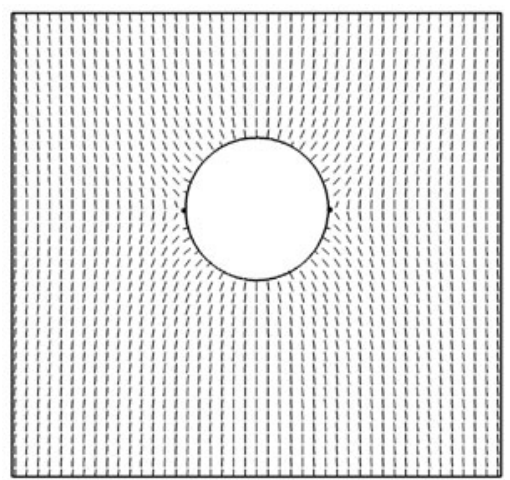

Figure 3. Defect configurations near a drop with homeotropic anchoring. (a) The satellite point defect, indicated by the black dot, within the $\boldsymbol{n}$ field. $A_{\sigma}=0.05, A_{K}=100$. $(b)$ The finite-element mesh for $(a)$ is refined around the interface and the satellite defect. $(c)$ Drawing of the director field for a Saturn-ring defect. $(d)$ The surface ring defect for $A_{\sigma}=0.05$, $A_{K}=100$, indicated by black dots on the equator of the drop.

not distinguish $\boldsymbol{n}$ and $\boldsymbol{- n}$. As a consequence, our vector-based theory cannot allow defect lines of half-strength; the surrounding $\boldsymbol{n}$ field inevitably contains an apparent discontinuity between $\boldsymbol{n}$ and $\boldsymbol{- n}$ and thus incurs an infinite elastic energy. Instead of the Saturn-ring defect with a strength of $-1 / 2$, therefore, we predict a surface ring as shown in figure $3(d)$. With this caveat, we reproduce all the features noted above, including the effect of $A_{K}$ on defect stability. In particular, for sufficiently strong anchoring, the satellite defect is produced if the initial $\boldsymbol{n}$ is radial near the drop surface, while the surface ring arises from an initially uniform $\boldsymbol{n}$ field. An additional parameter,

$$
A_{\sigma}=\frac{W}{\sigma},
$$

governs the shape of the drop, and a small value is used in most of the simulations to ensure a nearly spherical drop. Figure 4 plots the position of the satellite point defect as a function of $A_{K}$. The increase of anchoring energy moves the point defect farther from the drop, as is noted by Ruhwandl \& Terentjev (1997). At the limit of $A_{K} \rightarrow \infty$, $r_{d} / a \rightarrow 1.35$, which agrees well with prior calculations $\left(r_{d} / a=1.26\right.$ by Lubensky et al. 1998 and $\sqrt{2}$ by Pettey et al. 1998) and measurement $\left(r_{d} / a=1.4 \pm 0.1\right.$ by 


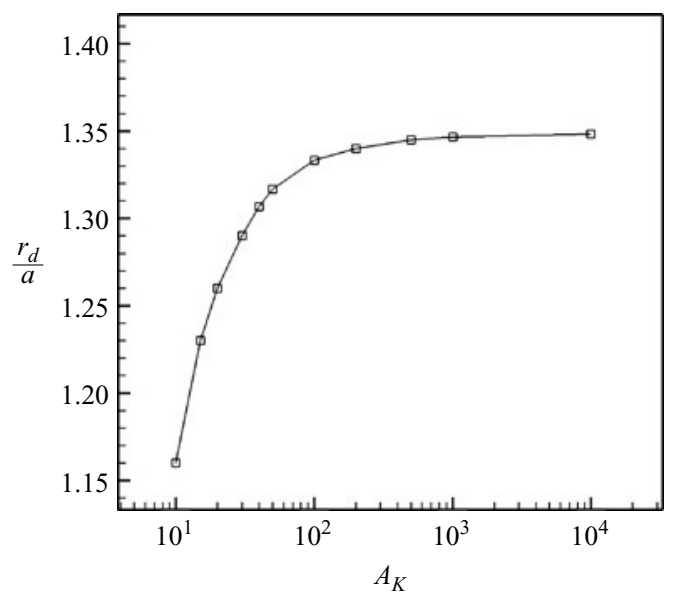

FIGURE 4. Position of the satellite point defect near a stationary drop with homeotropic anchoring. $r_{d}$ is the distance between the defect core and the centroid of the drop. For $A_{K}<10$, the point defect loses stability and gives way to a surface ring on the equator. $A_{\sigma}=0.05$.

Cluzeau et al. 2001). With decreasing $A_{K}$, the defect approaches the drop and causes a protrusion on the interface. As $A_{K}$ falls below a threshold value, around 10 in this case, the point defect opens up into a surface ring. This threshold $A_{K}$ is close to the previous Monte Carlo prediction of approximately 7 (Ruhwandl \& Terentjev 1997). The distance $r_{d}$ has some practical implications. Potentially it can be used as a measurement of the anchoring strength $W$, which is otherwise difficult to determine. Furthermore, $r_{d}$ also determines the particle spacing in self-assembled arrays of droplets in nematic emulsions (Poulin \& Weitz 1998).

\subsection{Flow-induced transformation of defect configuration}

Static defects may be driven from one configuration to the other by an external electric or magnetic field (Terentjev 1995; Loudet \& Poulin 2001). It will be interesting to see whether similar transitions can be effected by the flow surrounding a rising drop. Figure 5 shows schematically the computational domain for simulating rising drops in a nematic medium. A spherical drop of radius $a$ is initially centred at $(0,4 a)$, with either homeotropic or planar anchoring, although the latter will not be discussed until the next subsection. The far-field director orientation is vertical and parallel to the drop motion. A horizontal far-field $\boldsymbol{n}$ would upset axisymmetry and require a fully three-dimensional simulation. We also disallow azimuthal components of $\boldsymbol{n}$ and $\boldsymbol{v}$. Thus, $\boldsymbol{n}=(0,1)$ on the bottom, side and top walls. The velocity vanishes on the bottom and sidewalls, but the top is assigned a stress-free condition. On the axis of symmetry, we require $\partial / \partial r=0$ for all variables except the radial components of $\boldsymbol{n}$ and $v: n_{r}=0$ and $v_{r}=0$. For numerical parameters, the capillary width $\epsilon=0.01 a$, and a small grid size $h_{1}=0.006 a$ is used on the interface and near the defect (cf. figure $3 b$ ). Inside the drop and in the matrix, the grid sizes are $h_{2}=0.08 a$ and $h_{3}=0.1 a$, respectively. These prescribed values are guidelines for mesh generation, and the actual mesh is spatially unstructured and varies adaptively during the simulation. 


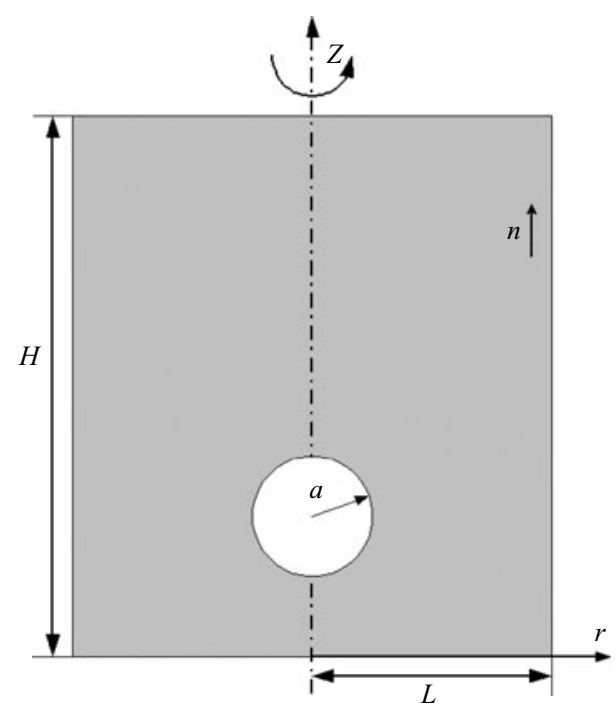

Figure 5. Computational domain for a Newtonian drop rising in a nematic whose far-field orientation is vertical. The geometry is axisymmetric and only half of the meridian plane is used in the computation. The drawing is not to scale; $H=20 a$ and $L=10 a$.

The rise of drops or bubbles in a nematic fluid is governed by 10 dimensionless numbers:

$$
\begin{aligned}
\alpha & =\frac{\rho_{2}}{\rho_{1}} \quad(\text { drop-to-matrix density ratio), } \\
\beta & =\frac{\mu}{\bar{\eta}} \quad(\text { drop-to-matrix viscosity ratio), } \\
E o & =\frac{\Delta \rho g a^{2}}{\sigma} \quad \text { (Eótvös number), } \\
M o & =\frac{\Delta \rho g \bar{\eta}^{4}}{\rho_{1}^{2} \sigma^{3}} \quad \text { (Morton number), }
\end{aligned}
$$

plus the 4 ratios of the 5 independent Leslie viscosities and the static parameters $A_{\sigma}$ and $A_{K}$. In $E o$ and $M o, \Delta \rho=\rho_{1}-\rho_{2}$. Drop and bubble shapes deviate from the spherical at large Eo (Grace, Wairegi \& Nguyen 1976), and our code has been shown to accurately capture this effect for Newtonian fluids (Yue et al. 2006b). For all results presented hereafter, $\alpha=0.5$ and $\beta=0.514$. A set of Leslie coefficients based on those of PAA (Chandrasekhar 1992) and MBBA (de Gennes \& Prost 1993) are chosen as the baseline. Values of $\alpha_{1}, \alpha_{3}, \alpha_{4}$ and $\alpha_{5}$ are fixed at the ratio of $\alpha_{1}: \alpha_{3}: \alpha_{4}: \alpha_{5}=5:-1: 18: 18$. This also fixes the characteristic viscosity $\bar{\eta}=\left(\alpha_{3}+\alpha_{4}+\alpha_{5}\right) / 2$. Then the viscosity ratio $\eta_{1} / \eta_{2}$ is varied through $\alpha_{2}$ (and $\alpha_{6}$ according to the Onsager relationship) to probe the effect of viscous anisotropy. Based on the terminal velocity $U$ of the rising drop, we determine the steady-state Reynolds number $R e=\rho_{1} U a / \bar{\eta}$ and Ericksen number $E r=\bar{\eta} U a / K$.

Our results show that the flow shifts the orientation field downstream, and modifies the relative stability of the ring and point defects. The 'phase diagram' in figure 6 depicts the stability of each configuration near a steadily rising drop, parametrized by $E r$ and $A_{K}$ that denote respectively the strengths of flow and surface anchoring as compared with the bulk elasticity. Six zones may be identified with different defect 


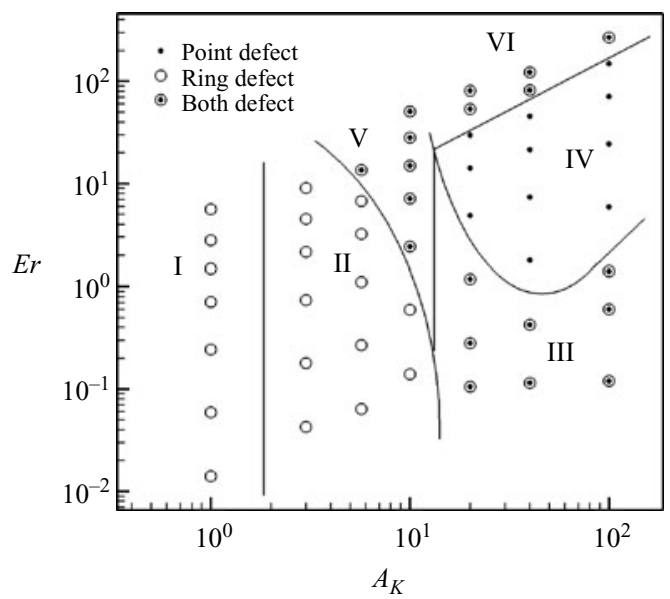

Figure 6. A 'phase diagram' of steady-state defect configurations. $A_{K}$ and $E r$ are varied by tuning $K$ and $g$, respectively. $A_{\sigma}=0.01, \eta_{1} / \eta_{2}=3$.

configurations. In zone I, the drop has an imaginary ring inside but no defects outside. In zone II, the surface ring is the sole stable configuration, while in zone IV, only point defects occur. In zone III, V and VI, both the surface ring and the satellite point defect are locally stable and either may appear depending on initial conditions.

For vanishing $E r$, the transitions from zone I to II and III with increasing anchoring strength are well known from static studies (Kuksenok et al. 1996; Ruhwandl \& Terentjev 1997). This simple picture holds up to $E r \sim 1$. In this weak-flow regime, the only flow effect is to shift the surface ring or satellite defect downstream. The shift is more pronounced for higher $A_{K}$, since stronger surface anchoring favours a smaller ring. For higher $E r$, a transition from zone II to $\mathrm{V}$ or from zone III to IV takes place depending on $A_{K}$. In zone $\mathrm{V}$, the point defect becomes locally stable; an initial point defect can now be stabilized in the wake of the drop by a sufficiently strong flow whereas in zone II, it would have opened up into a ring defect. If the initial condition has a ring defect, it remains stable in zone $\mathrm{V}$ but shifts downstream and shrinks in radius with increasing $E r$.

Going from zone III to IV with increasing Er, the ring defect loses stability. On a drop that initially bears a surface ring, the flow sweeps the ring downstream on the drop as it rises. If its terminal velocity puts it in zone IV, the surface ring will be shed into a satellite point defect in the wake. Starting with an initial point defect, we have only simulated the configuration with the defect in the wake of the drop. Having a point defect upstream of the drop appears unlikely in reality and may indeed be unstable to three-dimensional disturbances. Throughout zone III and zone IV, the point defect remains stable and shifts downstream with increasing $E r$. The steady-state position of the ring and point defects is shown in figure 7 as a function of $\mathrm{Er}$ that crosses from zone III to IV.

Increasing $E r$ further from zone IV, there is another transition to zone VI where the surface ring regains stability. At such high $E r$, the drop assumes an oblate shape, and an initial equatorial ring turns into a small surface ring near the bottom that cannot be shed into the wake as a point defect. Figure 8 shows an example of the steady-state director field in zone VI. Thanks to the oblate shape, the flow near the rear stagnation point of the drop is much reduced as compared with zone IV. The viscous forces are thus much weaker and can no longer drive the surface ring off. Similar configurations 


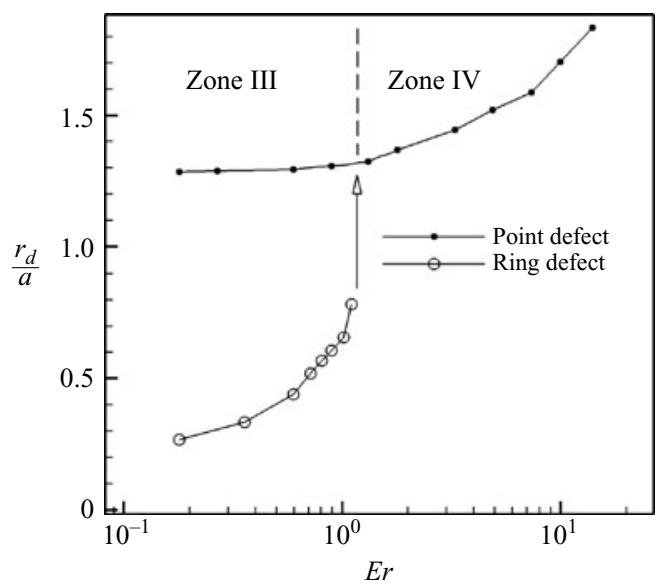

FIGURE 7. Steady-state position of the defect near a rising drop as a function of the Ericksen number. $r_{d}$ is the distance between the centroid of the drop and the point defect $\left(r_{d} / a>1\right)$ or the centre of the surface ring $\left(r_{d} / a<1\right)$. The arrow indicates the transition from zone III to IV at $E r=1.10$ when the surface ring defect gives way to a point defect. $A_{\sigma}=0.01, A_{K}=30$, $\eta_{1} / \eta_{2}=3$.

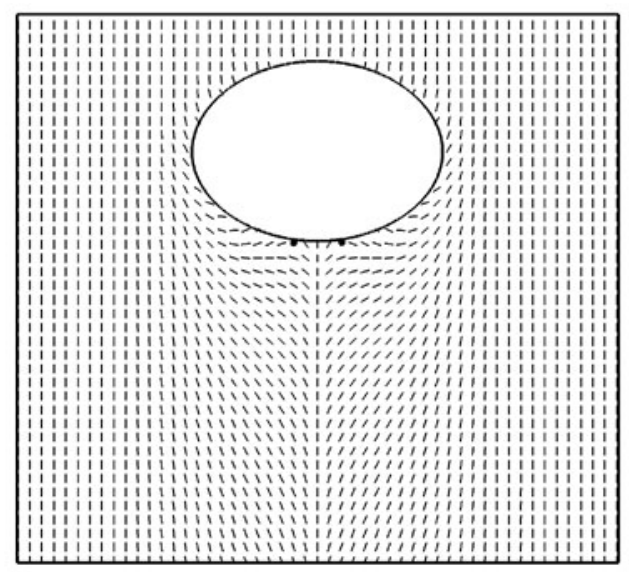

FIGURE 8. Director orientation around a steadily rising oblate drop in zone VI, with a small surface ring indicated by two black dots in the rear of the drop. $\eta_{1} / \eta_{2}=3, A_{\sigma}=0.01$, $A_{K}=20, E r=53.6, E o=1.20, M o=1.22 \times 10^{-6}$ and $\operatorname{Re}=21.9$.

occur at high $E r$ in zone V. In fact, zone V and VI are connected at the top, and their division is mostly a result of our describing the phase diagram in terms of increasing Er from equilibrium. The multiplicity of defect configurations in zones III, V and VI implies hysteresis. For example, raising $E r$ transforms a ring in zone III into a point defect in IV. Upon lowering $E r$ back into zone III, however, the point defect remains (cf. figure 7).

The trend in figure 7, showing defects being 'convected' downstream by flow, agrees with the results of Yoneya et al. (2005) and Fukuda et al. (2004), but contradicts the prediction of Stark \& Ventzki (2002) that defects shifts upstream under flow. As defects are orientation patterns rather than material properties, the convective effect is not intuitively obvious. Thus, we designed an experiment using silicone oil 
(a)

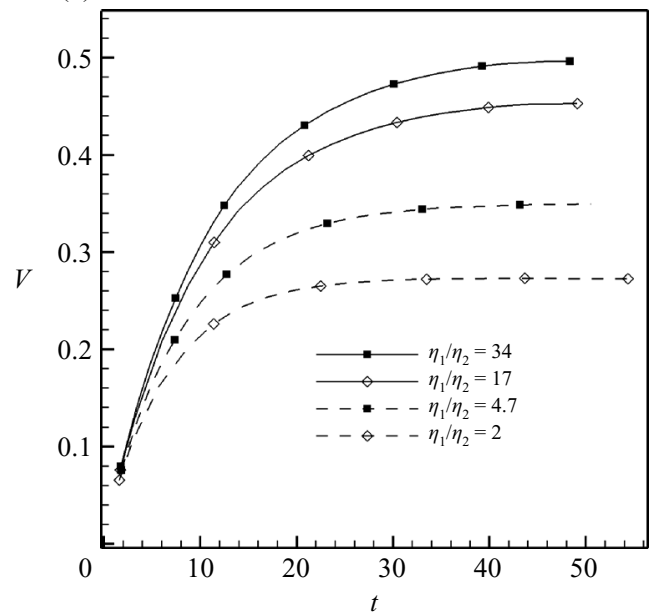

(b)

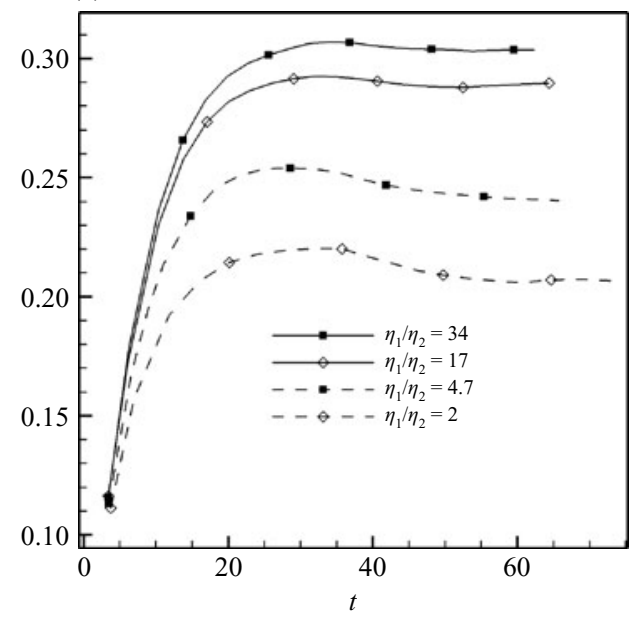

FigURE 9. Transient rising velocity of a drop in a nematic liquid crystal with $(a)$ planar anchoring and $(b)$ homeotropic anchoring. $E o=0.3, M o=5.56 \times 10^{-5}, A_{\sigma}=0.2$ and $A_{K}=40$. Time is made dimensionless by $\bar{\eta} /(\Delta \rho g a)$, and velocity by $\Delta \rho g a^{2} / \bar{\eta}$.

drops rising in the nematic 5CB (Khullar, Zhou \& Feng 2007). Both the Saturn ring and the point defect shift downstream as the rising velocity increases. At sufficiently high speed, the Saturn ring is shed into the wake as a point defect. This settles the question of the direction of convection, and confirms the flow-induced ring-to-point transformation predicted here. The experimental conditions correspond to $A_{K} \approx 25$ and $E r \approx 0.25$, comparable to the values in figure 7 . Note that Yoneya et al. (2005) have predicted a similar transformation at $E r \sim 10$, but with the flow field prescribed as the Stokes solution and with rigid anchoring $\left(A_{K} \rightarrow \infty\right)$.

We should mention that the ranges of dimensionless parameters in the preceding discussion correspond to common small-molecule nematics under reasonable flow conditions, and the same is true for the next section. For example, the Leslie viscosity ratios are close to those of flow-aligning nematics such as PAA and MBBA. Take MBBA (de Gennes \& Prost 1993): $\bar{\eta}=7.25 \times 10^{-2}$ Pas, $K=5 \times 10^{-12} \mathrm{~N}$ (average of the three elastic constants for splay, twist and bend). Then the range of $0.1 \lesssim E r \lesssim 100$ in figures 6 and 7 corresponds to rising velocities from 0.14 to $140 \mu \mathrm{m} \mathrm{s}^{-1}$ for a drop of diameter $100 \mu \mathrm{m}$, which are consistent with the experimental values of Khullar et al. (2007).

\subsection{Rising velocity, drag force and the flow field}

In this subsection, we investigate the effect of the nematic microstructure on the flow field, with special attention to the implications of the viscous anisotropy and defect configuration. The geometric setup of the computation is the same as in the last subsection ( $c f$. figure 5), but some of the parameter values differ. In particular, we will focus on a range of rise velocity that corresponds to zone IV for homeotropic anchoring, with the satellite defect being the sole stable configuration. This range displays the most interesting behaviour when a ring defect transforms into a satellite during the rise of the drop. Planar anchoring will be considered as well. Figure 9 shows the transient rising velocity of a drop with planar and homeotropic anchoring. To give a sense of the time and velocity scales, a silicone oil drop $100 \mu \mathrm{m}$ in diameter rising in MBBA would have $\bar{\eta} /(\Delta \rho g a)=1.54 \mathrm{~s}$ and $\Delta \rho g a^{2} / \bar{\eta}=32.5 \mu \mathrm{m} \mathrm{s}^{-1}$, both 
(a)

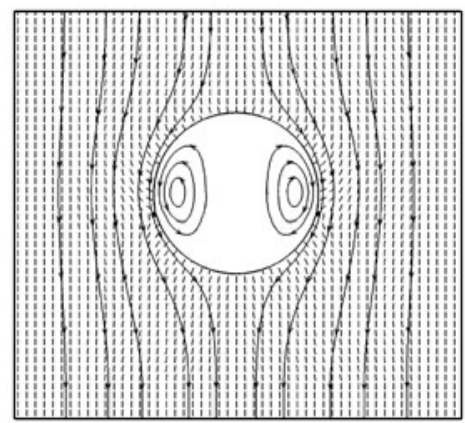

(c)

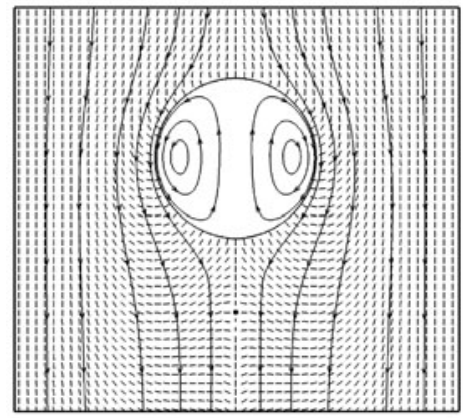

(b)

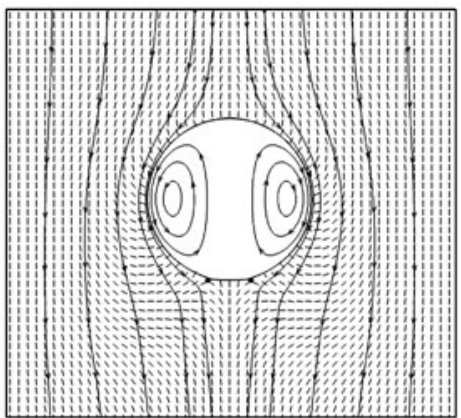

(d)

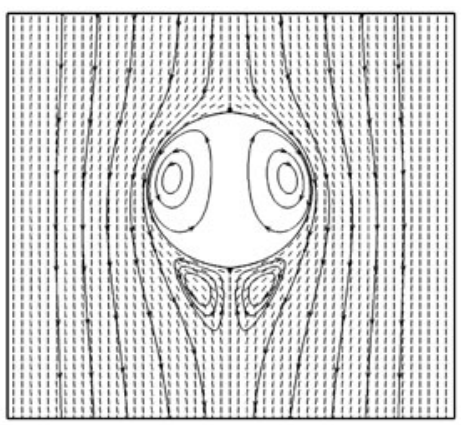

FIGURE 10. Director and flow fields around the drop with homeotropic anchoring at three times: $(a) t=6.33,(b) t=28.4$, and (c) final steady state at $t=52.7$, with $E r=15.8$ and $R e=6.43$. (d) The steady state for a drop with planar anchoring; $E r=25.2$ and $R e=10.3$. These correspond to the curves in figure 9 with $\eta_{1} / \eta_{2}=34$. For the streamlines, the reference frame is affixed to the centroid of the drop.

within the experimental range of Khullar et al. (2007). Within each plot, we examine the effects of viscous anisotropy by varying the ratio of two Miesowicz viscosities. As explained before, this is achieved by varying $\alpha_{2}$ and $\alpha_{6}$ while keeping the characteristic viscosity $\bar{\eta}=\left(\eta_{1}+\eta_{2}\right) / 2$ and the other Leslie coefficients fixed.

With planar anchoring, the rising velocity $V$ increases monotonically in time toward the terminal velocity $U$. For homeotropic anchoring, on the other hand, $V$ experiences an overshoot. This is caused by the transition from a surface ring to a point defect as explained in the last subsection. Figure 10 shows the director and flow fields near the drop with homeotropic anchoring at three times. Initially, $\boldsymbol{n}$ is vertical throughout the domain. A surface ring forms quickly on the equator of the drop and shifts downstream as the drop rises $(t=6.33)$. With the drop accelerating, the flow sweeps the defect ring towards the rear of the drop $(t=28.4)$, and eventually transforms it into a point defect as $V$ attains the terminal velocity $(t=52.7)$. Comparing figures $10(b)$ and $10(c)$, the $\boldsymbol{n}$ field with the point defect has a larger area - including the wake - in which $\boldsymbol{n}$ are nearly orthogonal to the streamlines. According to (2.14), the nematic exhibits higher viscosity there than in areas where $\boldsymbol{n}$ is aligned to the flow. Thus, the transformation from surface ring to point defect increases the viscous dissipation in the entire domain and thus the drag on the drop. This explains the overshoot in figure $9(b)$. Our recent experiment has confirmed such an overshoot during the ring-to-point defect transformation (Khullar et al. 2007). If the drop has a point defect at the start, or if the steady state falls in zones III, V or VI where the surface ring is stable, there will be no overshoot in $V$. For planar anchoring, two 


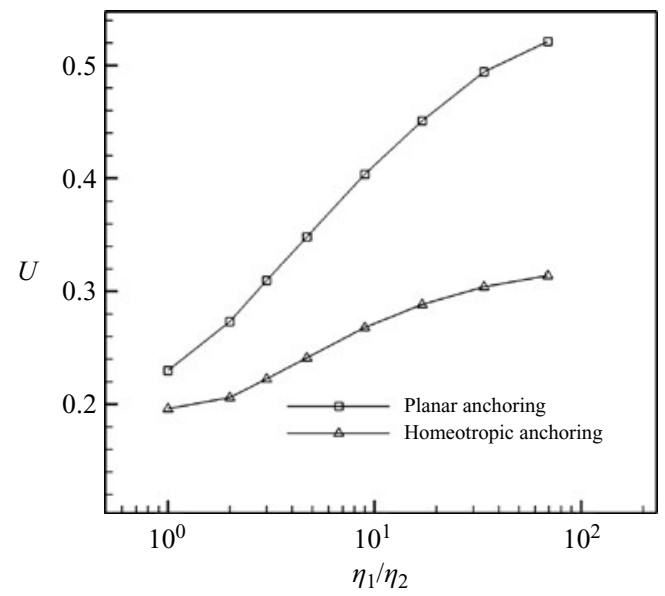

FIGURE 11. Terminal velocity $U$ of the drops in figure 9 as affected by viscous anisotropy. $U$ is made dimensionless by $\Delta \rho g a^{2} / \bar{\eta}$. The range of $U$ corresponds to $4.93<R e<10.9$ and $12.1<E r<26.8$ for planar anchoring, and $4.29<\operatorname{Re}<6.64$ and $10.5<E r<16.3$ for homeotropic anchoring.

boojums stay at the poles throughout the rise (figure $10 \mathrm{~d}$ ), and the rising velocity again shows no overshoot. Roughly speaking, the orientation distortion extends into the nematic bulk for a fraction of the drop diameter; within this region the flow is affected by the anisotropic viscosity.

With either type of anchoring, the rise velocity increases with viscous anisotropy as measured by $\eta_{1} / \eta_{2}$. Figure 11 gives a clearer view of this effect in terms of the terminal velocity $U$. Furthermore, planar anchoring produces a higher $U$ than homeotropic anchoring under otherwise identical conditions. This difference can again be explained by the drag as affected by different director fields. With planar anchoring (figure $10 d$ ), $\boldsymbol{n}$ aligns with the streamlines in most of the domain, apparently minimizing the total dissipation (Jadżyn \& Czechowski 2001). This leads to a smaller drag and hence a greater $U$ than the drop with homeotropic anchoring. In both cases, however, alignment between $\boldsymbol{n}$ and $\boldsymbol{v}$ is more prevalent throughout the domain than their being orthogonal, and larger areas experience $\eta_{2}$ than $\eta_{1}$. With increasing $\eta_{1} / \eta_{2}$, therefore, the overall viscous dissipation diminishes with $\eta_{2}$ and the rising velocity $U$ increases as in figure 11, and more significantly for planar anchoring. In fact, for each value of $\eta_{1} / \eta_{2}$, the $U$ values for both anchoring types are bounded by the Hadamard-Rybczynski predictions using $\eta_{1}$ and $\eta_{2}$.

The eddies in the wake of the drop in figure $10(d)$ form a vortex ring. It is not expected for Newtonian fluids at $R e=10.3$ and viscosity ratio $\beta=0.514$ (Dandy \& Leal 1989), nor does it appear for homeotropic anchoring. The explanation seems to rest with the anisotropic viscosity. The Reynolds number cited above is defined using the characteristic viscosity $\bar{\eta}$. In figure $10(d)$, the streamlines align with the director $\boldsymbol{n}$ to varying degrees in the flow field. Thus, the local viscosity may be much below $\bar{\eta}$ in some regions. In particular, the streamlines next to the drop surface follow the $\boldsymbol{n}$ field precisely. If we take the Miesowicz viscosity $\eta_{2}$ to be the local viscosity, the local Reynolds number will be around 180 . The high momentum of this layer of fluid then leads to flow separation in the wake. In contrast, homeotropic anchoring causes $\boldsymbol{n}$ to be mostly orthogonal to $\boldsymbol{v}$ near the drop surface. The local Reynolds number is much lower and no separation occurs. We have also noticed that for planar anchoring, 


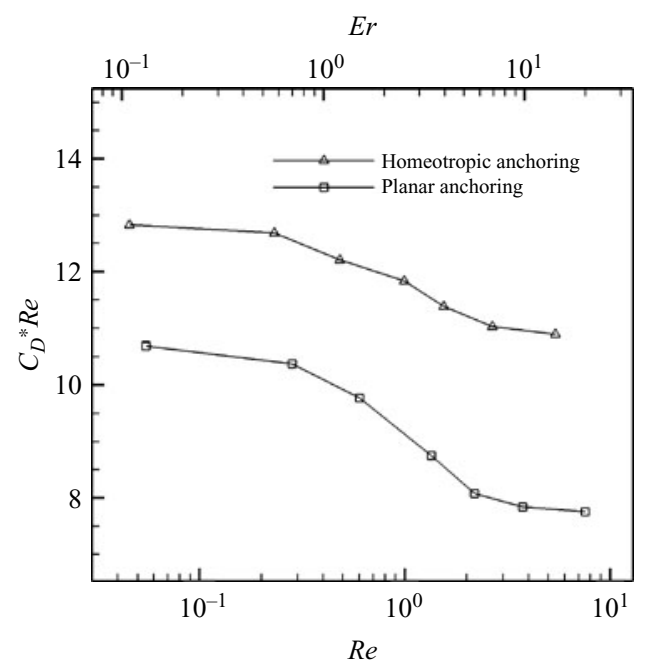

FIGURE 12. The drag coefficient for drops rising in a nematic as a function of $R e$ or $E r$. For homeotropic anchoring, the data correspond to the satellite configuration. $\eta_{1} / \eta_{2}=3, A_{\sigma}=0.2$, $A_{K}=40$.

the recirculating zone shrinks with decreasing $\eta_{1} / \eta_{2}$ and disappears altogether when $\eta_{1}=\eta_{2}$. This is consistent with viscous anisotropy being the cause of the vortex ring.

The drag on the drop can be further analysed in terms of the drag coefficient

$$
C_{D}=\frac{\frac{4}{3} \pi \Delta \rho g a^{3}}{\frac{1}{2} \rho_{1} U^{2}\left(\pi a^{2}\right)}
$$

defined from the terminal velocity $U$. The Hadamard-Rybczynski formula gives $C_{D} R e=8(1+1.5 \beta) /(1+\beta)$ for a spherical Newtonian drop moving in a Newtonian matrix at vanishing $R e$ (Batchelor 1980). In view of this formula, we plot the product $C_{D} R e$ against $R e$ and $E r$ in figure 12. $U$ and hence $R e$ and $E r$ are varied through the buoyancy force while keeping the viscosity ratio $\beta$ fixed. As noted before, homeotropic anchoring gives a higher drag than planar anchoring. If the matrix were a Newtonian fluid, $C_{D} R e$ would be constant for small $R e$ and increase with $R e$ for finite inertia. That $C_{D} R e$ decreases with increasing Reynolds number reflects the enhanced alignment of $\boldsymbol{n}$ by the flow field. This is better illustrated by $E r$ marked on the upper abscissa. For small Ericksen numbers, say $E r<1$, the director orientation is hardly affected by the surrounding flow. Thus $C_{D} R e$ remains roughly constant. As $E r$ exceeds unity, viscous flow effects become comparable to the elastic effects and the flow-alignment of $\boldsymbol{n}$ reduces $C_{D} R e$. This decline eventually levels off as the flow-alignment saturates around $E r=10$.

All prior results in the literature on the drag force are for rigid spheres with homeotropic anchoring at $R e=0$. Nevertheless, a comparison is interesting. In the limit of vanishing $E r$, Stark \& Ventzki (2001) fixed the $\boldsymbol{n}(\boldsymbol{r})$ field to the equilibrium solution with a point defect, and computed the drag in terms of an effective viscosity $\eta_{\text {eff }}$ defined from the Stokes formula. In our case, a similar $\eta_{\text {eff }}$ can be estimated from the Hadamard-Rybczynski formula. At the lowest $E r=0.109$ for homeotropic anchoring, our data give $\eta_{\text {eff }} / \eta_{2}=2.63$, which is comparable to the results of Stark $\&$ Ventzki (2001): 1.83 for MBBA and 2.32 for 5CB. Our somewhat larger drag may 
(a)

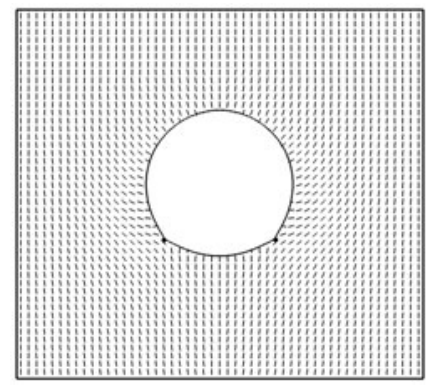

(b)

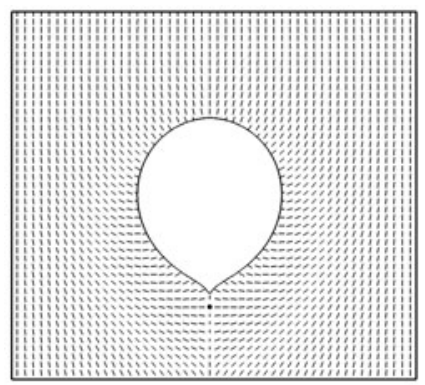

(c)

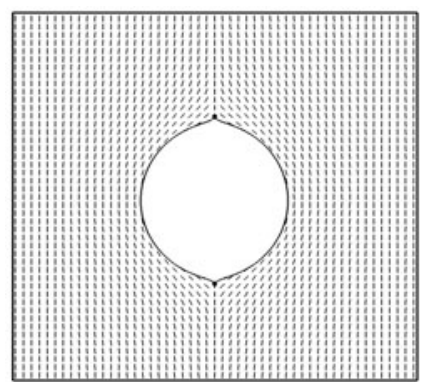

FIGURE 13. Non-spherical drop shapes produced by the nearby defects. Eo = 0.3, $M o=5.56 \times 10^{-5}, A_{\sigma}=0.5, A_{K}=15, \eta_{1} / \eta_{2}=4.7$. (a) A drop with homeotropic anchoring and a surface ring rising at $R e=5.54, E r=2.04$. (b) A drop with homeotropic anchoring and a point defect. $R e=3.92, E r=1.44$. (c) A drop with planar anchoring and boojums. $R e=7.16, E r=2.64$. The boojums give the highest rising velocity while the point defect the lowest.

have to do with wall confinement in the geometric setup (figure 5). The decrease of $\eta_{\text {eff }}$ with $\mathrm{Er}$ is consistent with the findings of Stark \& Ventzki (2002). At the highest $E r=14.1$ in figure $12, \eta_{\text {eff }} / \eta_{2}=2.22$. Although the Hadamard-Rybczynski formula no longer applies exactly at the finite $R e$ in this case, $\eta_{\text {eff }}$ asymptoting to a value significantly larger than $\eta_{2}$ bespeaks the 'orientation boundary layer' on the drop surface due to the homeotropic anchoring ( $c f$. figure 10c), inside which there is still considerable misalignment between $\boldsymbol{n}$ and $\boldsymbol{v}$. With even higher $E r$, the experiment of White et al. (1977) suggests that $\eta_{\text {eff }}$ approaches $\eta_{2}$. For planar anchoring, our data give $\eta_{\text {eff }} / \eta_{2}=2.18$ and 1.60 for the low-Er and high-Er limits of figure 12 .

So far we have used relatively large values for the interfacial tension $\sigma$ (or small values of $A_{\sigma}$ ) to keep the surface curvature of the drop smooth. At larger $A_{\sigma}$, drops deform in response to the nearby orientational field, especially the presence of defects. Figure 13 illustrates three typical situations with the surface ring, satellite and boojum defects. The proximity of defects causes large curvature on the drop surface as a result of the competition between interfacial tension and anchoring energy. The cost in anchoring energy due to the defects is reduced at the expense of interfacial area such that the total energy is minimized. The lemon shape in figure $13(c)$ is well known in nematic drops with planar anchoring, and has also been reported for isotropic drops in nematic medium (Nastishin et al. 2005; Zhou et al. 2007). The drop with boojums rises the fastest while that with the satellite the slowest.

\section{Summary}

This paper presents a computational study of the rise of a Newtonian drop in a nematic liquid crystal. The problem is a rough counterpart of the HadamardRybczynski problem in Newtonian fluids, although the Reynolds number ranges up to about 10 and mild drop deformation occurs. The key physics revealed by the simulation is the two-way coupling between the flow field and the molecular orientation field, and especially the configuration of orientational defects. The results can thus be summarized as follows.

(a) Effect of flow on the orientational field. With either a satellite point defect or a surface ring defect, the flow sweeps the defect downstream. Thus, the surface ring shrinks and moves toward the rear stagnation point, and at sufficiently high 
Ericksen number may be transformed into a point defect. An initial point defect moves farther downstream with increasing Ericksen number. The stability of the two defect configurations is depicted by a phase diagram in terms of the Ericksen number and the ratio between surface anchoring and bulk elastic energies.

(b) Effect of orientation on the flow field. This is mainly manifested through the viscous anisotropy of the fluid. Drops with planar anchoring rise faster than those with homeotropic anchoring since the director field is better aligned with the streamlines. With homeotropic anchoring, a drop experiences an overshoot in the transient rising velocity when a ring defect changes into a detached point defect. With both types of anchoring, the drag coefficient decreases with the Ericksen number because stronger viscous flow aligns the director to the streamline and reduces frictional dissipation.

Through a systematic examination of the coupling between flow and molecular orientation, we strive to construct a coherent picture for the fluid mechanics of a particle moving in a nematic liquid. So far, the predicted flow effects on defect convection and transformation have been verified experimentally, as has the overshoot in rise velocity accompanying the defect transformation (Khullar et al. 2007). Finally, we point out two limitations in our work. The first is the vectorial nature of the LeslieEricksen theory. The original version cannot handle defects as they would constitute singularities. A relaxation of the unit-length requirement on the director allows integer-strength defects to be simulated, but the Saturn ring has to be represented by a surface ring. The latter has fewer degrees of freedom and possibly different stability regimes from an unattached Saturn ring. This restriction can be removed by adopting a tensorial representation of the molecular orientation (Rey \& Tsuji 1998; Feng et al. 2000; Yoneya et al. 2005). Second, the axisymmetric two-dimensional geometry of the computation precludes the interesting scenario of drops rising in a nematic with horizontal far-field orientation.

Acknowledgement is made to the Donors of The Petroleum Research Fund, administered by the American Chemical Society, for partial support of this research. J.J.F. was also supported by the NSERC, the Canada Research Chair program, the Canada Foundation for Innovation and the NSFC (Grant no. 50390095). C.Z. acknowledges partial support by a University Graduate Fellowship from UBC.

\section{REFERENCES}

Akers, B. \& Belmonte, A. 2006 Impact dynamics of a solid sphere falling into a viscoelastic micellar fluid. J. Non-Newtonian Fluid Mech. 135, 97-108.

Batchelor, G. K. 1980 An Introduction to Fluid Mechanics. Cambridge University Press.

CARLSSON, T. 1984 Theoretical investigation of the shear flow of nematic liquid crystals with the Leslie viscosity $\alpha_{3}>0$ : Hydrodynamic analogue of first order phase transitions. Mol. Cryst. Liq. Cryst. 104, 307-334.

Chandrasekhar, S. 1992 Liquid Crystals, 2nd edition. Cambridge University Press.

Cluzeau, P., Poulin, P., Joly, G. \& Nguyen, H. T. 2001 Interactions between colloidal inclusions in two-dimensional smetic-c" films. Phys. Rev. E 63, 031702.

Dandy, D. S. \& LeaL, L. G. 1989 Buoyancy-driven motion of a deformable drop through a quiescent liquid at intermediate Reynolds numbers. J. Fluid Mech. 208, 161-192.

Diogo, A. C. 1983 Friction drag on a sphere moving in a nematic liquid crystal. Mol. Cryst. Liq. Cryst. 100, 153-165.

Feng, J. J., Liu, C., Shen, J. \& Yue, P. 2005 An energetic variational formulation with phase field methods for interfacial dynamics of complex fluids: Advantages and challenges. In Modeling of Soft Matter (ed. M.-C. T. Calderer \& E. Terentjev). Springer. 
Feng, J. J., Sgalari, G. \& Leal, L. G. 2000 A theory for flowing nematic polymers with orientational distortion. J. Rheol. 44, 1085-1101.

FenG, J. J. \& Zhou, C. 2004 Orientational defects near colloidal particles in a nematic liquid crystal. J. Colloid Interface Sci. 269, 72-78.

Fukuda, J.-I., Stark, H., Yoneya, M. \& Yokoyama, H. 2004 Dynamics of a nematic liquid crystal around a spherical particle. J. Phys.: Condens. Matter 16, S1957-S1968.

De Gennes, P. G. \& Prost, J. 1993 The Physics of Liquid Crystals. Oxford University Press.

Grace, J. R., Wairegi, T. \& NGUYen, T. H. 1976 Shapes and velocities of single drops and bubbles moving freely through immiscible liquids. Trans. Inst. Chem. Engrs 54, 167-173.

Gu, Y. \& Аввотт, N. L. 2000 Observation of Saturn-ring defects around solid microspheres in nematic liquid crystals. Phys. Rev. Lett. 85, 4719-4722.

JACQMIN, D. 1999 Calculation of two-phase Navier-Stokes flows using phase-field modelling. J. Comput. Phys. 155, 96-127.

JADŻYN, J. \& CZECHOWSKI, G. 2001 The shear viscosity minimum of freely flowing nematic liquid crystals. J. Phys.: Condens. Matter 13, L261-L265.

Khullar, S., Zhou, C. \& Feng, J. J. 2007 Dynamic evolution of topological defects around drops and bubbles rising in a nematic liquid crystal. Phys. Rev. Lett. (submitted).

KlÉMAn, M. 1983 Points, Lines and Walls: In Liquid Crystals, Magnetic Systems and Various Ordered Media. Wiley.

Kuksenok, O. V., Ruhwandl, R. W., ShiyanovskiI, S. V. \& Terentjev, E. M. 1996 Director structure around a colloid particle in a nematic liquid crystal. Phys. Rev. E 54, 5198-5204.

Kuss, E. 1978 pVT-data and viscosity-pressure behavior of MBBA and EBBA. Mol. Cryst. Liq. Cryst. 47, 71-83.

LaVRentovich, O. D. 1998 Topological defects in dispersed liquid crystals, or words and worlds around liquid crystal drops. Liq. Cryst. 24, 117-125.

Leslie, F. M. 1968 Some constitutive equations for liquid crystals. Arch. Rat. Mech. Anal. 28, 265-283.

LiU, C. \& Shen, J. 2003 A phase field model for the mixture of two incompressible fluids and its approximation by a Fourier-spectral method. Physica D 179, 211-228.

Liu, C. \& Walkington, N. J. 2000 Approximation of liquid crystal flows. SIAM J. Numer. Anal. 37, 725-741.

Loudet, J. C., Barois, P. \& Poulin, P. 2000 Colloidal ordering from phase separation in a liquid crystalline continuous phase. Nature 407, 611-613.

Loudet, J. C. \& Poulin, P. 2001 Application of an electric field to colloidal particles suspended in a liquid-crystal solvent. Phys. Rev. Lett. 87, 165503.

Lubensky, T. C., Pettey, D., Currier, N. \& Stark, H. 1998 Topological defects and interactions in nematic emulsions. Phys. Rev. E 57, 610-625.

Mondain-Monval, O., Dedieu, J. C., Gulik-Krzywicki, T. \& Poulin, P. 1999 Weak surface energy in nematic dispersions: Saturn ring defects and quadrupolar interactions. Eur. Phys. J. B 12, $167-170$.

Nastishin, Y. A., Liu, H., Schneider, T., Nazarenko, V., Vasyuta, R., Shiyanovskit, S. V. \& LAVRentovich, O. D. 2005 Optical characterization of the nematic lyotropic chromonic liquid crystals: Light absorption, birefringence, and scalar order parameter. Phys. Rev. E 72, 041711.

Poulin, P., Cabuil, V. \& Weitz, D. A. 1997 a Direct measurement of colloidal forces in an anisotropic solvent. Phys. Rev. Lett. 79, 4862-4865.

Poulin, P., Stark, H., Lubensky, T. C. \& Weitz, D. A. $1997 b$ Novel colloidal interactions in anisotropic fluids. Science $\mathbf{2 7 5}, 1770-1773$.

Poulin, P. \& Weitz, D. A. 1998 Inverted and multiple nematic emulsions. Phys. Rev. E 57, 626-637.

Rapini, A. \& Papoular, M. 1969 Distortion d'une lamelle nematique sous champ magnetique: Conditions d'ancrage aux parois. J. Phys. (Paris) C 30, 54-56.

ReY, A. D. \& TsujI, T. 1998 Recent advances in theoretical liquid crystal rheology. Macromol. Theory Simul. 7, 623-639.

Ruhwandl, R. W. \& Terentjev, E. M. 1996 Friction drag on a particle moving in a nematic liquid crystal. Phys. Rev. E 54, 5204-5210.

Ruhwandl, R. W. \& Terentuev, E. M. 1997 Monte Carlo simulation of topological defects in the nematic liquid crystal matrix around a spherical colloidal particle. Phys. Rev. E 56, 5561-5565. 
StARK, H. 1999 Director field configurations around a spherical particle in a nematic liquid crystal. Eur. Phys. J. B 10, 311-321.

Stark, H. 2001 Physics of colloidal dispersions in nematic liquid crystals. Phys. Rep. 35, 387-474.

Stark, H. \& VentzKi, D. 2001 Stokes drag of spherical particles in a nematic environment at low Ericksen number. Phys. Rev. E 64, 031711.

Stark, H. \& Ventzki, D. 2002 Non-linear stokes drag of spherical particles in a nematic solvent. Europhys. Lett. 57, 60-66.

Terentjev, E. M. 1995 Disclination loops, standing alone and around solid particles, in nematic liquid crystals. Phys. Rev. E 51, 1330-1337.

Tixier, T., Heppenstall-Butler, M. \& Terentjev, E. M. 2006 Spontaneous size selection in cholesteric and nematic emulsions. Langmuir 22, 2365-2370.

Trebin, H. R. 1982 The topology of nonuniform media in condensed matter physics. Adv. Phys. 31, $195-254$.

White, A. E., Cladis, P. E. \& Torza, S. 1977 Study of liquid crystals in flow. Part I. conventional viscometry and density measurements. Mol. Cryst. Liq. Cryst. 43, 13-31.

Yамамото, R. 2001 Simulating particle dispersions in nematic liquid-crystal solvents. Phys. Rev. Lett. 87, 075502.

Yoneya, M., Fukuda, J.-I., Yokoyama, H. \& Stark, H. 2005 Effect of a hydrodynamic flow on the orientation profiles of a nematic liquid crystal around a spherical particle. Mol. Cryst. Liq. Cryst. 435, 75-85.

Yue, P., Feng, J. J., Liu, C. \& Shen, J. 2004 A diffuse-interface method for simulating two-phase flows of complex fluids. J. Fluid Mech. 515, 293-317.

Yue, P., Feng, J. J., Liu, C. \& Shen, J. 2005a Diffuse-interface simulations of drop coalescence and retraction in viscoelastic fluids. J. Non-Newtonian Fluid Mech. 129, 163-176.

Yue, P., Feng, J. J., Liu, C. \& Shen, J. $2005 b$ Interfacial force and Marangoni flow on a nematic drop retracting in an isotropic fluid. J. Colloid Interface Sci. 290, 281-288.

Yue, P., Feng, J. J., Liu, C. \& Shen, J. 2005c Transient drop deformation upon startup of shear in viscoelastic fluids. Phys. Fluids 17, 123101.

Yue, P., Feng, J. J., Liu, C. \& Shen, J. 2005d Viscoelastic effects on drop deformation in steady shear. J. Fluid Mech. 540, 427-437.

Yue, P., Zhou, C. \& Feng, J. J. $2006 a$ A computational study of the coalescence between a drop and an interface in Newtonian and viscoelastic fluids. Phys. Fluids 18, 102102.

Yue, P., Zhou, C. \& Feng, J. J. 2007 Spontaneous shrinkage of drops and mass conservation in phase-field simulations. J. Comput. Phys. 223, 1-9.

Yue, P., Zhou, C., Feng, J. J., Ollivier-Gooch, C. F. \& Hu, H. H. $2006 b$ Phase-field simulations of interfacial dynamics in viscoelastic fluids using finite elements with adaptive meshing. J. Comput. Phys. 219, 47-67.

Zhou, C., Yue, P. \& Feng, J. J. 2006 Formation of simple and compound drops in microfluidics devices. Phys. Fluids 18, 092105.

Zhou, C., Yue, P., Feng, J. J., Liu, C. \& Shen, J. 2007 Heart-shaped bubbles rising in anisotropic liquids. Phys. Fluids 19, 041703. 